\title{
EELS in the TEM: nanometer resolution without a nanoprobe
}

\author{
P. SCHATTSCHNEIDER, C. TISCHLER ${ }^{*}$ and H. BANGERT ${ }^{*}$
}

Laboratoire de Mécanique, Sols, Structures et Matériaux, Ecole Centrale Paris, 92295 Châtenay-Malabry, France

${ }^{*}$ Institut für Angewandte und Technische Physik der Technischen Universität Wien, 1040 Wien, Austria

\begin{abstract}
With many TEMs, the resolution of electron energy loss spectrometry (EELS) is limited to $\sim 100 \mathrm{~nm}$ since a finer probe cannot be positioned and maintained accurately enough for the long dwell times necessary. The alternative TEM image mode (i. e. projecting the area of interest onto the spectrometer aperture) suffers from the energy dependent chromatic blurring, limiting the resolution also to several $100 \mathrm{~nm}$. Due to inevitable misalignment of post specimen lenses (PSLs), electrons that have suffered a characteristic loss will hit the screen at a different position than the elastically scattered ones, even when the chromatic blurring is compensated by objective lens defocus. We describe a correction method for both the chromatic blurring and the chromatic image shift. It is shown by example that quantitative EELS analysis can be done on a scale of better than $10 \mathrm{~nm}$ with spots of arbitrary size in regular TEM image-mode.
\end{abstract}

In EELS, elemental analysis with high spatial resolution is usually done by focussing a fine probe onto the area to be analysed. With many TEMs, the resolution of this method is limited to some $100 \mathrm{~mm}$ since a finer probe cannot be positioned and maintained accurately enough for the long dwell times necessary for EELS analysis. The alternative TEM image mode (i. e. projecting the area of interest onto the spectrometer aperture) suffers from two shortcomings:

1. Without compensating measures, the chromatic aberration of the post specimen lenses (PSLs) limits the resolution to several $100 \mathrm{~nm}$, depending on experimental conditions and the chosen energy loss $E$, (normally a ionisation edge). When $C_{c}$ is known, this can be compensated by a proper underfocus of the objective lens

$$
\Delta f=C_{c} \frac{E}{E_{0}}
$$

See table 1 for typical values.

2. Due to inevitable misalignment of PSLs, inelastically scattered electrons will hit the screen at a different position than the elastically scattered ones, even when the chromatic blurring is compensated by objective lens defocus. (Proper defocus of all PSLs would remove that problem but is impractical). In fig. 1, the chromatic image shift is explained by exaggerating the amount of tilt and shift of the optical axes of two consecutive lenses. When the elastic image coincides with the spectrometer aperture (point $\mathrm{E}$ ), the image made up of inelastically scattered electrons (I) is above the screen since the focal widths of all PSLs are smaller. Compensation of chromatic blurring by OL underfocus brings the image back to the screen ( $\left.I^{\prime}\right)$.

From the construction it is clear that the line $\overline{\mathrm{EI}}$ is the axis of the convergent ray bundle when the voltage center is properly corrected. It follows that the defocussed elastic image is centrally superimposed on the refocussed inelastic image (which cannot be observed on the screen) when 


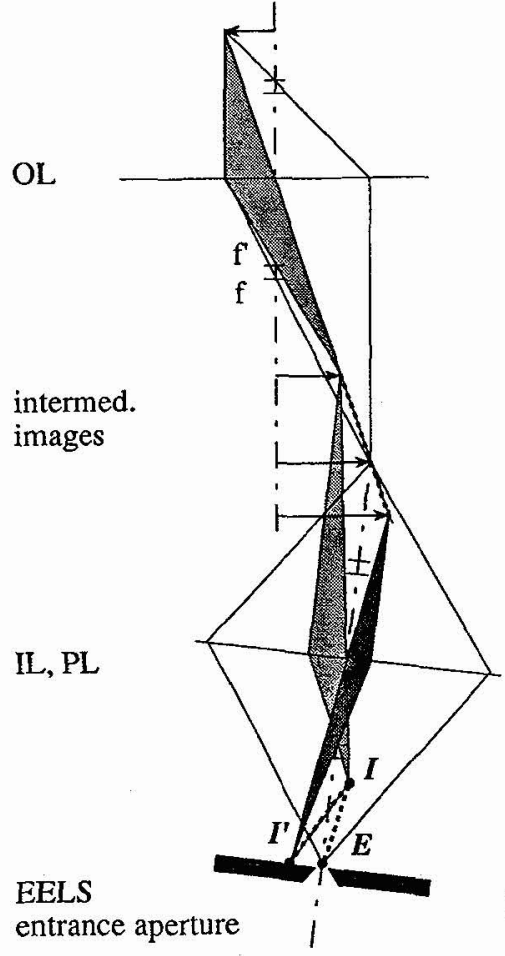

Tab. 1: Radius of aberration disk for $C_{c}=6.6 \mathrm{~mm}$ and $\theta=13.6 \mathrm{mrad}$.

\begin{tabular}{|lccc|}
\hline edge & $\mathrm{E}(\mathrm{eV})$ & $\Delta \mathrm{f}(\mu)$ & $\mathrm{r}(\mathrm{nm})$ \\
\hline $\mathrm{C}-\mathrm{K}$ & 285 & 9.4 & 127 \\
$\mathrm{Sn}-\mathrm{M}$ & 484 & 15.9 & 217 \\
$\mathrm{Al}-\mathrm{K}$ & 1560 & 51.5 & 700 \\
\hline
\end{tabular}

Fig. 1: Chromatic image shift caused by misalignment of PSLs. Tilt and shift of lens axes not to scale. Shaded: ray paths of inelastically scattered electrons. Dark shaded: refocussed inelastic image, refocussed by OL underfocus.

the voltage center is properly corrected. Thus, the chromatic image shift can be compensated by positioning the area to be analysed off-axis, after having determined the amount and direction of shift with a well visible image detail such as a hole or a protruding edge.

The method was applied to a sputtered AlSn specimen showing linelike structure of unknown origin-see fig. 2. The line distance was $a \approx 50 \mathrm{~nm}$. Spectra were taken on the lines and in between.

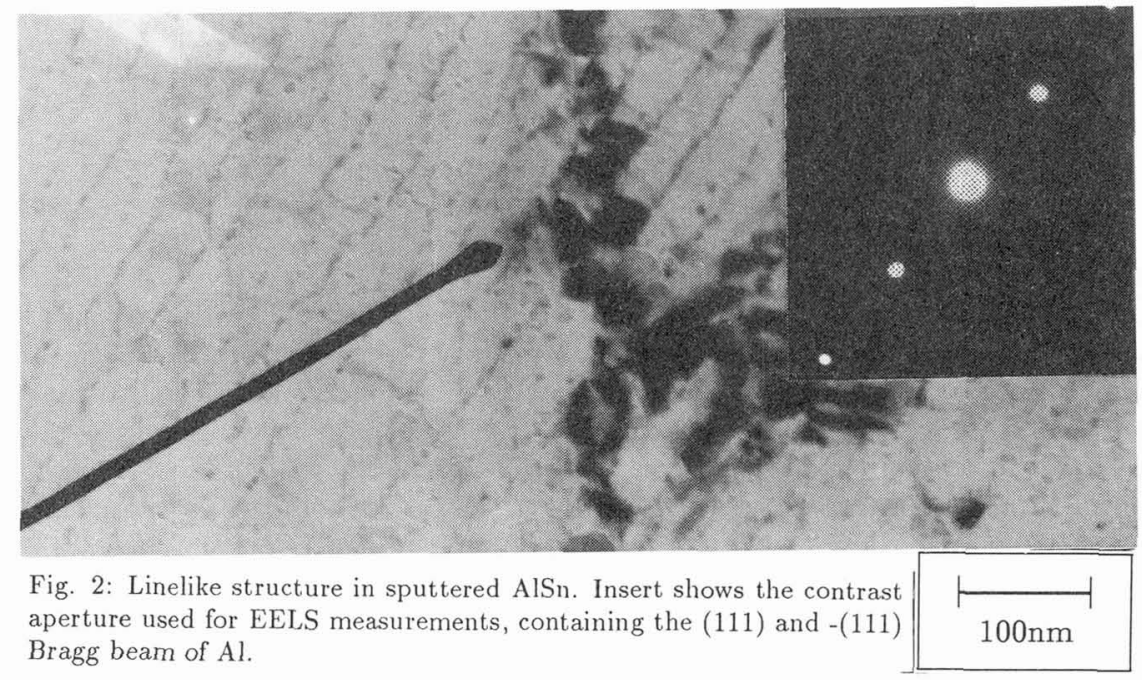


Assuming an idealized one-dimensional density distribution of constituent $j$ as a function of position $x$ on the specimen,

$$
n_{j}(x)=\hat{n}_{j}+\sum_{l} \delta(x-a l) \Delta_{j}
$$

where $\hat{n}_{j}$ is the atomic density between the lines (matrix density) and $\Delta_{j}$ is the increase present on the lines, one can calculate the measured intensity as

$$
I(E, x) \propto \sum_{j} \sigma_{j}(E)\left[\hat{n}_{j}+\Delta_{j} G(E, x)\right] .
$$

The partial ionisation cross sections $\sigma_{j}(E)$ can be calculated [1] or taken from a library [2]. The blurring function $G$ is an integral of the chromatically blurred image of the lines, performed over the spectrometer aperture positioned at $x$. It depends on the model distribution-see fig. 3. Note that the abszissa is in deviation from the energy loss at which the image is refocussed (in our case,

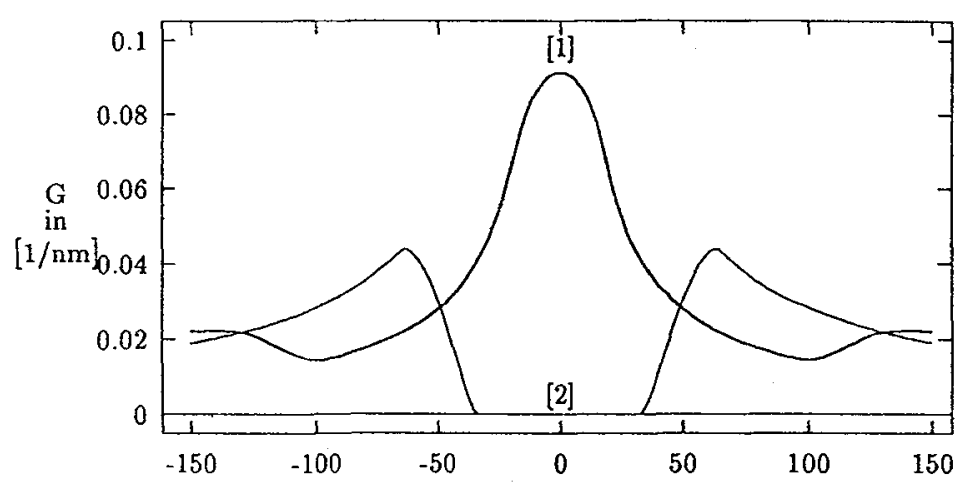

Fig. 3: Blurring function $G(E, x)$ for linelike structure. [1]: on the lines $(x=0)$; [2]: in between $(x=a / 2)$. Deviation from refocussed loss in $\mathrm{eV}$.

this is the oxygen K-edge at $532 \mathrm{eV}$ ). Observing that $G$ tends to a constant value for $E>100 \mathrm{eV}$, one obtains relative intensities by dividing eq. 3 by the intensity under the Al-K-edge (at $1560 \mathrm{eV}$ ):

$$
S(E, x)=\frac{\sigma_{S n}(E)}{\sigma_{A l}} \cdot\left[\hat{c}_{S n}+\Delta c_{S_{n}} G(E, x)\right]+\frac{\sigma_{O}(E)}{\sigma_{A l}} \cdot\left[\hat{c}_{O}+\Delta c_{O} G(E, x)\right]
$$

Here, we have assumed that oxygen and tin is present both in the matrix and on the lines. The atomic concentrations are defined relative to aluminium, $\hat{c}_{j}=\hat{n}_{j} / n_{A l}$ is the matrix concentration, and the increase on the lines is

$$
\Delta c_{j}=\frac{\Delta_{j}}{n_{A l}}=d \cdot \frac{\Delta n_{j, e f f}}{n_{A l}} .
$$

The concentration increase measured on the lines depends on the product of line width $d$ and the effective increase in atomic volume density $\Delta n_{j, e f f}$ for each constituent. This is true as long as the spectrometer aperture of $l=22 \mathrm{~nm}$ diameter is much larger than the line width (which was assumed to approach zero in the present model, eq. 2).

We obtained a mean relative atomic volume concentration of oxygen to aluminium of 0.575 between the lines, and 0.878 on the lines-see fig. 4. The detection of $\mathrm{Sn}$ is more difficult since the Sn-M edge at $484 \mathrm{eV}$ has a delayed maximum and overlaps the O-I edge at $532 \mathrm{eV}$. From fig. 3 we have that the spectrum of the matrix contains no line signal in the interval $[490 \mathrm{eV}, 570 \mathrm{eV}]$, thus, it is a pure $\mathrm{Sn}$-spectrum for $E<532 \mathrm{eV}$. This fact can be used to extract the Sn-contribution, using a standard spectrum. 
From high magnification images, the effective line width was found to be less than $5 \mathrm{~nm}$. Assuming $d=5 \mathrm{~nm}$, the Sn-concentration on the lines was calculated to be approximately 8 times higher than in between. Since the Sn-signal in the matrix is at the detection limit, the tin is predominantly present on the lines, possibly forming very thin platelike microprecipitates that constitute the lines. Thus the increased oxygen content on the lines is probably due to $\mathrm{SnO}$ or to $\mathrm{SnO}_{2}$. In order to decide the case, one may calculate the volume oxygen concentration of the unknown constituent as a function of $d$, from eq. 5 .

$$
\frac{n_{O, e f f}}{n_{A l}}=\Delta c_{O} \cdot \frac{1}{d}+\hat{c}_{O}
$$

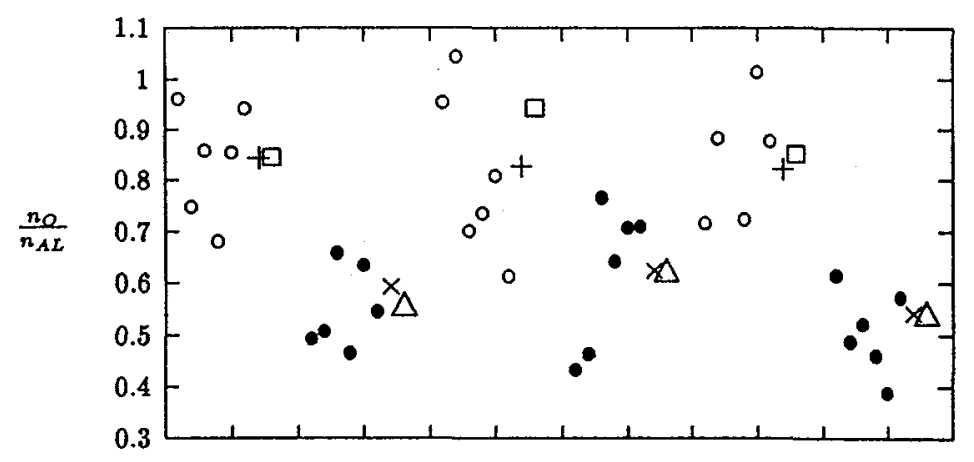

Fig. 4: Atomic concentration of oxygen in the matrix (•) and on the lines (o). Circles: single spectra; triangles, squares: averaged; crosses: averaged over selected spectra (no apparent drift). The abszissa is the number of spectra, from 1 to 60 .

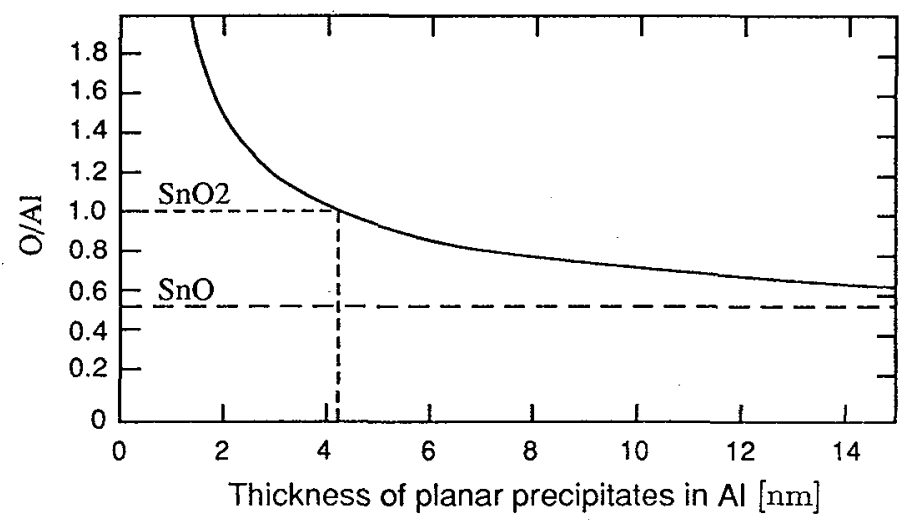

Fig. 5: Oxygen concentration $n_{O} / n_{A l}$ on the lines as a function of thickness of planar precipitate. Values for tin-oxide and-dioxide are also given.

The graph is given in fig. 5 , together with the expected values for the oxide and the dioxide. There is no value of $d$ consistent with $\mathrm{SnO}$. Assuming that the lines contain $\mathrm{SnO}_{2}$ yields a linewidth of $4.3 \mathrm{~nm}$, in good agreement with the estimate from the micrographs.

From the scatter in the data, fig. 4 , the minimum detectable linewidth is found to be $d_{\min } \approx 0.17 l$. In the present case, we have $d_{\min } \approx 2 \mathrm{~nm}$. This is the effective spatial resolution of the method.

\section{References}

[1] EGERTON R. F., Scanning El. Microsc. 1984/2, 505.

[2] GATAN Inc., EL/P Software. 